\title{
Erratum to: Non Existence of a Density Expansion of the Transport Coefficients in a Moderately Dense Gas
}

\author{
E.G.D. Cohen
}

Published online: 19 November 2009

(C) Springer Science+Business Media, LLC 2009

\section{Erratum to: J Stat Phys \\ DOI 10.1007/s10955-009-9838-6}

The complete correct sentences are:

1. On page 4, line 5:

"Finally the $J_{n}(f \ldots f)$ in (4) are integrals over infinite space over the positions and the velocities of all $(n-1)$ particles in the cluster, except over particle 1, and possess the cluster property i.e. each particle in the cluster must have at least one collision with another cluster particle (including particle 1) and in addition an irreducibility property of the collisions in the cluster is needed (cf. footnote 4 and $[14,15]$ ); if not, their contributions to $J_{n}$ vanish."

2. On page 4, line 14:

"It should be emphasized again that the structure of the $J_{n}$ is similar to that of the corresponding virial coefficients in equilibrium involving $n$ interacting particles, in that they have both the cluster property, i.e. they vanish unless all $n$ particles interact or collide at least once with another particle in the cluster and in addition an irreducibility property of the collisions in the cluster is needed (cf. footnote 4 and $[14,15])$."

Erratum should be Addendum: it contains an addition of a not printed but essential condition in two sentences on page 4, which have been added in the two corrected complete sentences above.

The online version of the original article can be found under doi:10.1007/s10955-009-9838-6.

E.G.D. Cohen $(\varangle)$

Laboratory of Statistical Physics, The Rockefeller University, 1230 York Avenue, New York, NY 10065, USA

e-mail: egdc@rockefeller.edu 